\title{
Treatment of hemobilia-induced biliary obstruction by transpapillary gallbladder and common bile duct drainage using three biliary winged stents
}

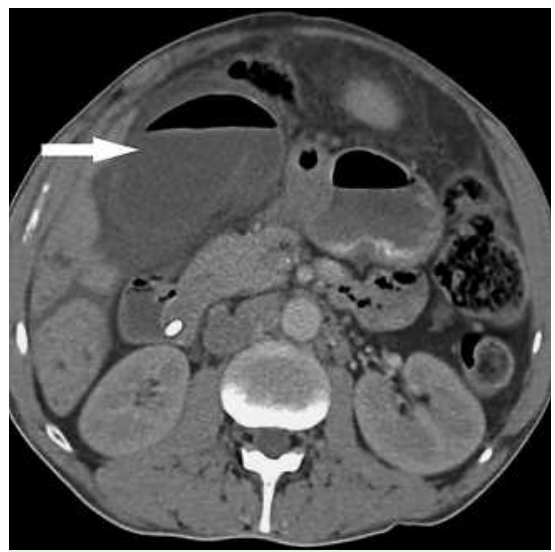

Fig. 1 Computed tomographic image showing a dilated gallbladder (arrow) with an airfluid level.

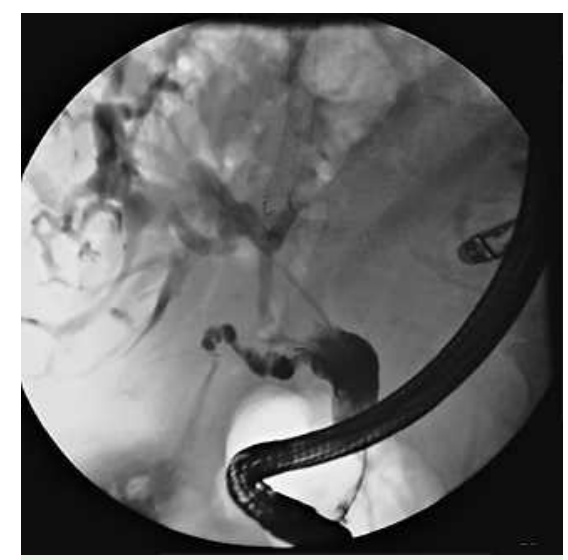

Fig. 2 Cholangiography demonstrated partial cystic duct obstruction and a markedly dilated distal common bile duct due to thrombus. The right-sided intrahepatic biliary tree is also dilated.

A 43-year-old man with Caroli's disease underwent a transjugular intrahepatic portosystemic shunt procedure, after which he developed marked hemobilia that was believed to be secondary to hepatic artery injury. He then developed acute cholangitis as a result of the increased pressure on the bile ducts from obstructing blood clots. Four separate endoscopic retrograde cholangiopancreatograms were performed to clear thrombus from his bile ducts and relieve the obstruction with conventional stent placement, but after dislodgment of the clots

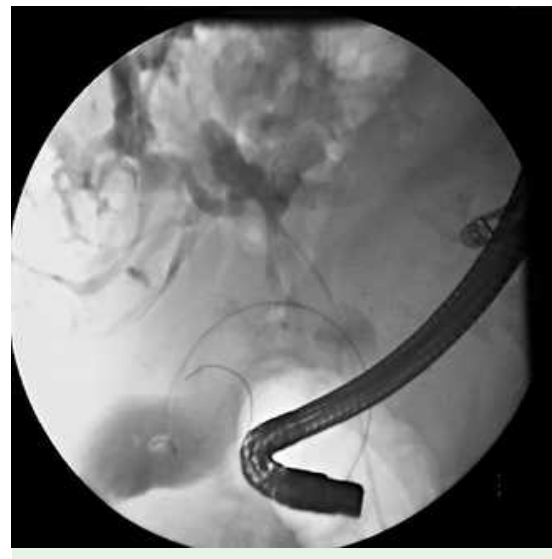

Fig. 3 The guide wire is seen here coursing through the cystic duct and curling within the gallbladder.

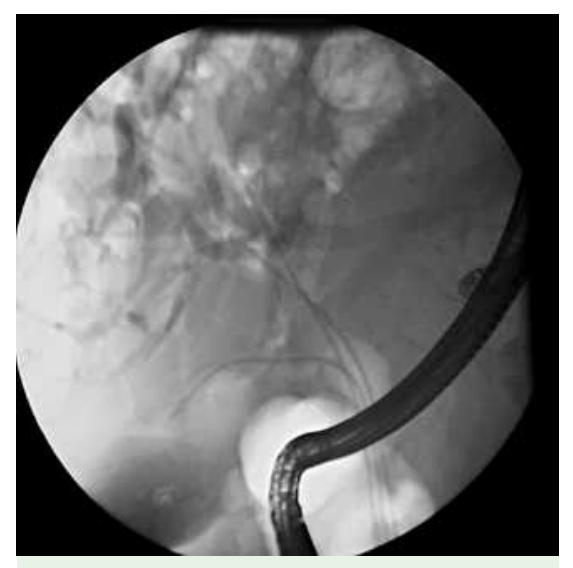

Fig. 4 The proximal end of the biliary wing stent can be seen here to be within the gallbladder lumen. Two additional wing stents were placed in branches of the right hepatic duct.

the hemobilia recurred each time, with reocclusion of the stent.

While he was in hospital he complained of acute-onset abdominal pain. Computed tomography showed blood within a dilated common bile duct, and an air-fluid level inside a distended gallbladder ( $\bullet$ Fig. 1). Repeat endoscopic retrograde cholangiopancreatography showed dilatation of the cystic duct and the common bile duct ( $\bullet$ Fig. 2). A 0.035 -inch guide wire was passed through the cystic duct into the gallbladder lumen ( $\bullet$ Fig.3), and a $10-\mathrm{Fr}, 15-\mathrm{cm}$, double-flapped biliary winged stent (ViaDuct Stents, GI Supply,
Camp Hill, Pennsylvania, USA) was inserted over the angled course of the wire ( Fig. 4). Two additional winged stents $(10 \mathrm{Fr}, 15 \mathrm{~cm}$ ) were positioned in branches of the right hepatic duct. His cholangitis subsequently resolved and the bleeding was treated with arterial embolization. The winged stent is a novel stent design that was introduced by Raju et al. [1] in 2006 as a pancreatic endoprosthesis. Unlike conventional stents, it allows for drainage around the outside of the prosthesis. It was developed to allow for unencumbered drainage of the pancreatic duct at all sites where secondary branches join the main duct. When used in the biliary tree, it is postulated that it facilitates improved drainage by limiting instant occlusion as bile is forced around the stent. The winged stent appeared to help in this case by enabling adequate drainage of bile and thrombus around the stent from all parts of the biliary tree. This resulted in reduced pain and resolution of the obstructive cholangitis.

Endoscopy_UCTN_Code_TTT_1AR_2AZ

Endoscopy_UCTN_Code_TTT_1AR_2AJ

\section{J. M. Buscaglia, K. R. Parashette, \\ P. I. Okolo III.}

Johns Hopkins University School of Medicine, Johns Hopkins Hospital, Baltimore, Maryland, USA

\section{References}

1 Raju GS, Gomez G, Xiao SY et al. Effect of a novel pancreatic stent design on shortterm pancreatic injury in a canine model. Endoscopy 2006; 38: 260-265

\section{Bibliography}

DoI $10.1055 / s-2007-967060$

Endoscopy 2008; 40: E58

(c) Georg Thieme Verlag KG Stuttgart · New York . ISSN 0013-726X

\section{Corresponding author}

\section{J. M. Buscaglia, MD}

Room 7100-A

Johns Hopkins Hospital

1830 E. Monument Street

Baltimore 21205

Maryland

USA

Fax: +1-410-955-2108

jbuscaglia@jhmi.edu 